\title{
Beam-transport optimization for cold-neutron spectrometer
}

\author{
Kenji Nakajima $^{1, a}$, Seiko Ohira-Kawamura ${ }^{1}$, Tatsuya Kikuchi ${ }^{1}$, Ryoichi Kajimoto ${ }^{1}$, Nobuaki Takahashi ${ }^{2}$, Mitsutaka \\ Nakamura $^{1}$, Kazuhiko Soyama ${ }^{1}$ and Toyotaka Osakabe ${ }^{3}$ \\ ${ }^{1}$ Materials \& Life Science Division, J-PARC Center, Tokai, Ibaraki 319-1195, Japan \\ ${ }^{2}$ Institute for Chemical Research, Kyoto University, Gokasho Uji-city, Kyoto, Japan 611-0011, Japan \\ ${ }^{3}$ Quantum Beam Science Directorate, Japan Atomic Energy Agency, Tokai, Ibaraki 319-1195, Japan
}

\begin{abstract}
We report the design of the beam-transport system (especially the vertical geometry) for a cold-neutron disk-chopper spectrometer AMATERAS at J-PARC. Based on the elliptical shape, which is one of the most effective geometries for a ballistic mirror, the design was optimized to obtain, at the sample position, a neutron beam with high flux without serious degrading in divergence and spacial homogeneity within the boundary conditions required from actual spectrometer construction. The optimum focal point was examined. An ideal elliptical shape was modified to reduce its height without serious loss of transmission. The final result was adapted to the construction requirements of AMATERAS. Although the ideas studied in this paper are considered for the AMATERAS case, they can be useful also to other spectrometers in similar situations.
\end{abstract}

\section{Introduction}

Recent progress in fabricating high-quality supermirrors has changed spectrometer design. One can easily decide to employ a long flight path length, for example, to realize lower background by using a curved guide or to get fine resolution which can be obtained by long flight time, without significant intensity loss. Actually, at J-PARC, one-third of the instruments at the Materials and Life Science Experimental Facility (MLF) have long ( $\geq 30 \mathrm{~m}$ ) beam transport sections [1].

The cold-neutron disk-chopper spectrometer AMATERAS [2] is one of the spectrometers at J-PARC with a long flight path: the distance from the moderator to the sample is $30 \mathrm{~m}$. AMATERAS is a direct-geometry spectrometer equipped with newly developed high-speed disk-choppers that serve as monochromating and pulseshaping choppers. The spectrometer is dedicated to inelastic and quasi-elastic neutron scattering from cold to subthermal energies. As expected for this type of instrument, high intensity and low background are critical to the performance of the spectrometer. Thus, the beam transport section is one of the key components of AMATERAS. Therefore, this section was extensively studied before finalizing the design.

In the beam-transport geometry of AMATERAS, we expect different roles for different directions. The horizontal geometry was considered to minimize the background. We decided to adopt a curved guide. The studies on the curved guide for AMATERAS were reported in a separate paper [3].

The vertical geometry was designed to maximize the intensity at the sample position. We considered that the most promising approach is to adopt an elliptically shaped mirror [4], in which, ideally, only a single reflection is

\footnotetext{
${ }^{a}$ Corresponding author: kenji.nakajima@j-parc.jp
}

required in transmission of neutrons from one focal point to the other. Extensive studies on neutron guide mirrors using Mote Carlo simulation revealed that it is no longer true when the source has finite size [5,6]. Nevertheless, elliptical mirrors are powerful. In our previous study [7], we examined the elliptical shape for AMATERAS and found that it offered significant advantages. Therefore, in our initial design, we used the elliptical geometry for the vertical shape of the AMATERAS beam-transport.

Next, we developed the actual design of the vertical beam-transport geometry and simultaneously started construction of the spectrometer. Then we faced a problem. In elliptical mirrors, the beam height can be very large at the midsection of the beam path. That causes unacceptable increase of the beam-transport cost. Another difficulty caused by excessive beam height is the required chopperslit size. To obtain high energy resolution, AMATERAS is equipped with three high-speed disk-choppers at $7.1 \mathrm{~m}$, $14.2 \mathrm{~m}$ and $28.4 \mathrm{~m}$ from the source (Fig. 1), which run at a maximum frequency of $f=350 \mathrm{~Hz}$. The rotation speed is almost at the limit obtainable by known technology. In particular, because the longer slits make a disk fragile, reducing the disk-slit heights is critical for the AMATERAS high-speed choppers. In turn, this criterion limits the beam height in the transport section.

In this study, we used Monte Carlo simulations to optimize the beam-transport design, especially with regard to the vertical geometry. The focal point and reduction of the beam height were examined. For the given boundary conditions, the mirror shape was optimized to maximize beam-transport performance.

\section{Initial conditions}

In the present study on the beam-transport system, the boundary conditions were as follows: the beam-transport 


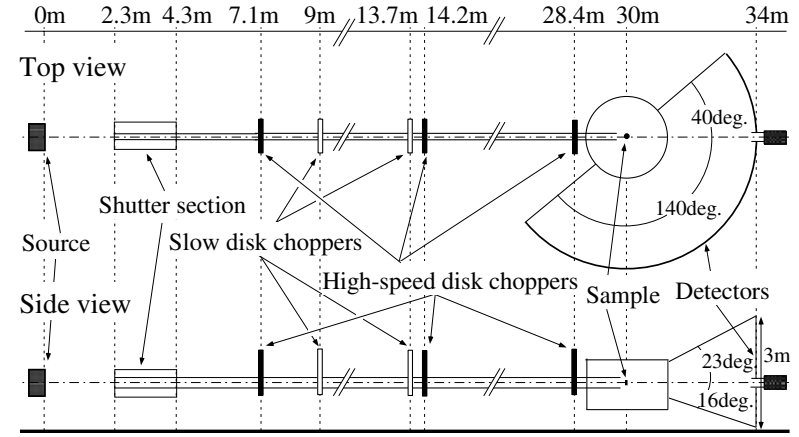

Figure 1. Overview of AMATERAS.

section that we considered extended between 2.3 and $30 \mathrm{~m}$ from the source. We could not modify the front end (i.e., 0 $2.3 \mathrm{~m}$ ). In our optimization, we ignored stainless steel and iron ducts (cross section: $102 \times 102 \mathrm{~mm}^{2}$ at the minimum) which are located there. Downstream of the duct was a shutter section that can accommodate a guide mirror of height of $90 \mathrm{~mm}$.

AMATERAS was designed for an incident neutron energy $E_{\mathrm{i}}$ of $1 \mathrm{meV}<E_{\mathrm{i}}<80 \mathrm{meV}$ and a relatively small sample size (10 $\mathrm{mm}$ wide and $20 \mathrm{~mm}$ high). The horizontal geometry was determined prior to fixing the vertical geometry. The horizontal dimension of the beam was $30 \mathrm{~mm}$ and a curved section with a radius of $2000 \mathrm{~m}$ began just after the first high-speed disk-chopper and continued to $26.8 \mathrm{~m}$ from the source. The horizontal geometry was included in all simulations presented here without mention.

A shutter section $(2.3-4.3 \mathrm{~m}$ from the source (Fig. 1)) was constructed prior to constructing AMATERAS. A guide tube $(30 \mathrm{~mm}$ width and $90 \mathrm{~mm}$ height $)$ with an $m=3$ mirror was already installed ( $m$ indexes the critical angle $\theta_{\mathrm{C}}$ of the supermirror: $\theta_{\mathrm{C}}=m \theta_{\mathrm{Ni}}$, where $\theta_{\mathrm{Ni}}$ is the critical angle of $\mathrm{Ni}$ ). In simulations shown in here, therefore, we fixed the value of $m$ to be 3 for this part.

All Monte Carlo simulations were performed with McStas [8]. Because we focused on performance in the beam-transport design and ignored the detailed intensity distribution caused by the actual source, we used Source. flat component as a neutron source.

\section{Vertical-geometry optimization}

\subsection{Focal point}

An ideal elliptical mirror focuses light from one focal point to the other. An ideal focal point has zero size. However, a real neutron source has finite size. For AMATERAS, the spectrometer views the coupled moderator, which is $100 \mathrm{~mm}$ in height. Problems caused by this finite source size can be avoided by setting the first focal point slightly behind the moderator [4], as also confirmed in our previous study of AMATERAS [7].

As the first step in optimizing the vertical geometry of the actual design, we tried to decide how far to place the focal point from the moderator. We tried several elliptical shapes, which were constrained by the following conditions: (i) the first focal point is $x_{\mathrm{f}} \mathrm{m}$ behind the source, and the second focal point is fixed at the sample

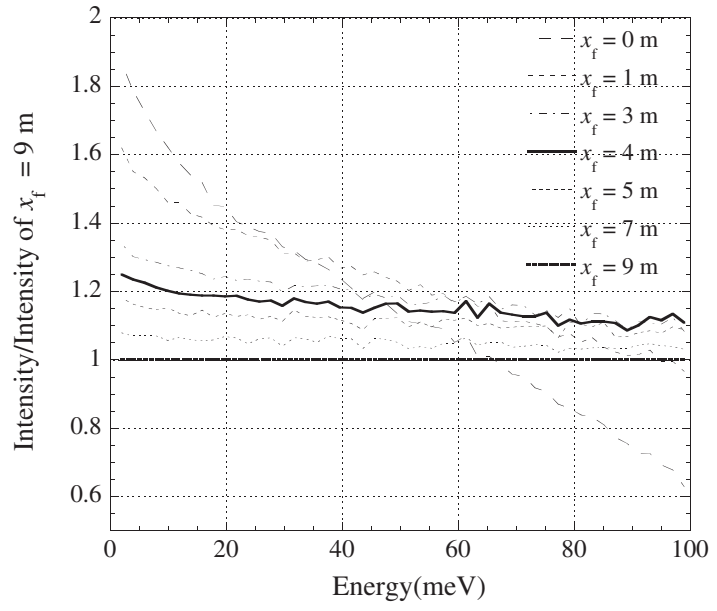

Figure 2. Energy dependencies of simulated neutron intensity of elliptical mirror guides with various $x_{\mathrm{f}} \mathrm{s}$, where $x_{\mathrm{f}}$ is the distance between focal point and source. Each intensity is integrated over a $10 \times 20 \mathrm{~mm}^{2}$ area at the sample position and is normalized by that of the elliptical mirror guide with $x_{\mathrm{f}}=9 \mathrm{~m}$.

position. (ii) The ellipsoid height is $90 \mathrm{~mm}$ at $2.3 \mathrm{~m}$ from the source.

We used Monte Carlo simulations to check the performance including the intensity, divergence, and spatial distribution at the sample position. Part of these results are shown in Fig. 2, which gives the intensity ratio, where intensity is defined as the number of neutrons integrated over a $10 \times 20 \mathrm{~mm}^{2}$ area at the sample position, and the ratio is formed by normalizing each intensity to that at $x_{\mathrm{f}}=9 \mathrm{~m}$. At $x_{\mathrm{f}}=0$, neutrons from the narrow area at the center of the source contribute to the majority of the intensity, while, with increasing $x_{\mathrm{f}}$, neutrons from the off-center area increase their relative populations at the sample position. Over the entire energy range of interest $(1 \mathrm{meV}<E<80 \mathrm{meV}$ ), a higher transmission is obtained as $x_{\mathrm{f}}$ decreases to $1 \mathrm{~m}$. However, we found that the inhomogeneity in spatial intensity distribution at the sample position could not be ignored for $x_{\mathrm{f}} \leq 3 \mathrm{~m}$; hence, we used $x_{\mathrm{f}}=4 \mathrm{~m}$.

\subsection{Height reduction and intensity}

As mentioned above, our main interest was to reduce the height of the beam-transport section without unacceptable reduction of the beam-transmission performance. To compromise between large cross sections of the beamtransport and small slits of choppers, a combination of a number of ballistic mirrors (so-called "eye-of-the-needle" geometry) [9] was proposed. The idea of a parabola feeder [6] is considered to be effective when a highspeed disk-chopper is located relatively far upstream in the beam-transport system. In the AMATERAS case, however, locations of three high-speed disk-choppers are spreaded over the middle part of the beam path. We were afraid that having many converging/diverging sections in the midsection of the beam-transport can be serious sources of transmission loss. Therefore, we decided to try to find another way. 
One of the simplest methods to achieve this goal is to use a spatulate ellipsoidal curve. For a point source surrounded by a mirror surface, transmission of the mirror does not depend on its height. (Here we consider 100\% reflectivity of the mirror for simplicity.) On the other hand, in reality, the source has a finite size, and the source and the sample are not surrounded by mirror surfaces. In such a case, a larger aperture of the inlet of the guide accepts larger number of neutrons. However, a large aperture causes the elliptical shape larger and, therefore, the height of the guide increases.

An alternative idea is to use an elliptical mirror as large as possible and cut the higher part by replacing it with a straight section. At the straight part in this modified geometry, the neutron beam hits the mirror at a steeper angle, and the ideal single-reflection trajectory no longer focuses on the focal point on the sample. The difference between reflection angles for straight lines and those for elliptical lines might be small, because curvatures of elliptical mirrors are small especially at the middle section in most cases. In addition, the finite source size is also expected to reduce the difference in performance between the two reflections.

Figure $3 \mathrm{a}$ shows the modified elliptical shape (lower panel) and the original elliptical shape (upper panel). In the modified shape, the part of the mirror above $90 \mathrm{~mm}$ is replaced with a straight mirror. The reflection angle viewed from the sample position as a function of distance from the source is shown in Fig. 3b. Figure $3 \mathrm{~b}$ also gives the supermirror critical angle for various values of $m$ and neutron energies. We planned to use $m=3$ and $m=4$ for the beam-transport mirror. In this constraint, we expected no serious degradation in beam properties even in the case of the modified elliptical mirror, because the reflection angle at most part of the mirror is smaller than the critical angles for $m=3$ or $m=4$. To confirm it, the transport performance of the modified elliptical mirror was studied by Monte Carlo simulations. The simulations discussed hereafter assumed an $m=4$ mirror except at the shutter section, where we assumed $m=3$. Figure $3 c$ shows the intensity in a $10 \times 20 \mathrm{~mm}^{2}$ area at the sample position as a function of energy. Intensity loss due to the modified elliptical mirror ranges from $4 \%$ (at $E=1 \mathrm{meV}$ ) to $8 \%$ (at $E=80 \mathrm{meV}$ ) of the intensity of the elliptical mirror which, as expected, is an acceptable result.

However, such an abrupt connection between the straight and elliptical geometries affects the beam divergence. As shown in Fig. 4, gaps appear in the divergence distribution, which results in an inhomogeneous spatialintensity distribution at the sample position. Gaps in the divergence distribution were also observed when we positioned the focal point closer to the source [7]. The divergence anomaly in this case, however, is caused by a discontinuous connection of different geometry mirrors as was reported on a similar truncated geometry in a former study [10]. Actually, the glancing angle from the sample area $(20 \mathrm{~mm}$ in height $)$ to the region connecting the straight and the elliptical sections is $0.3-0.5^{\circ}$, which coincides with the divergence gaps. (a)

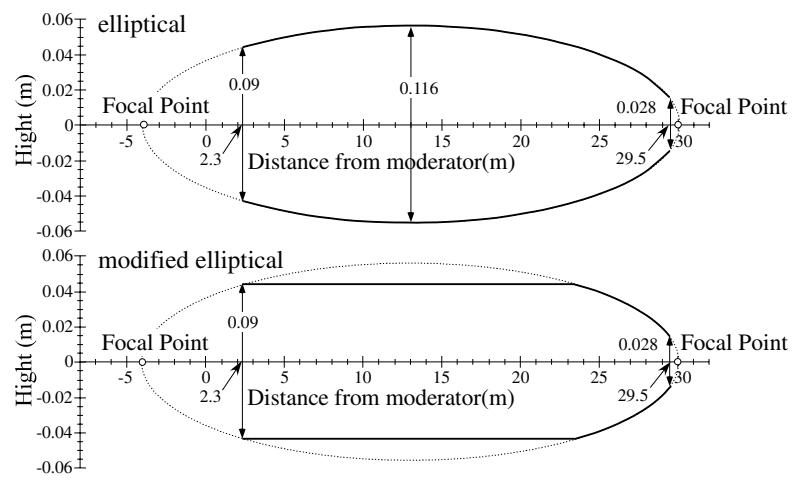

(b)

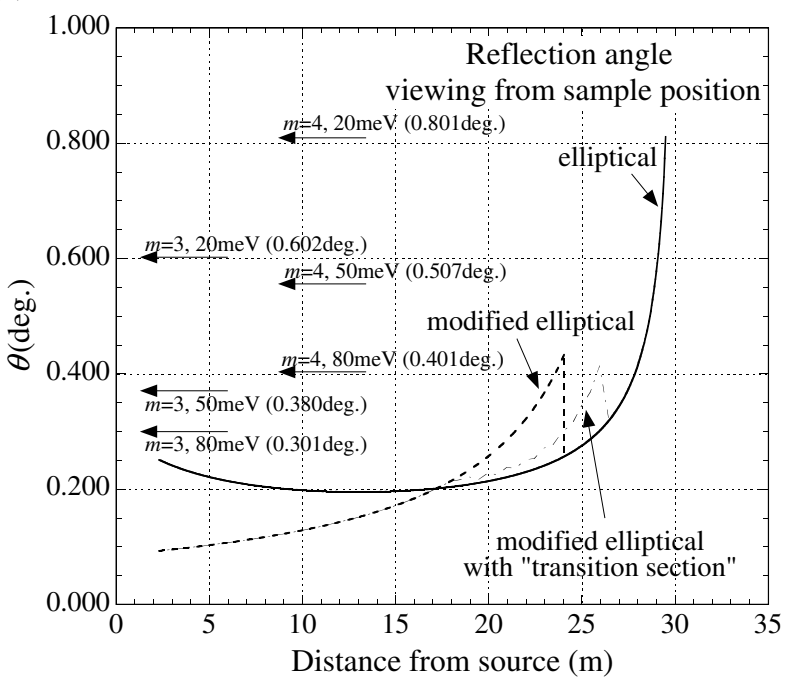

(c)

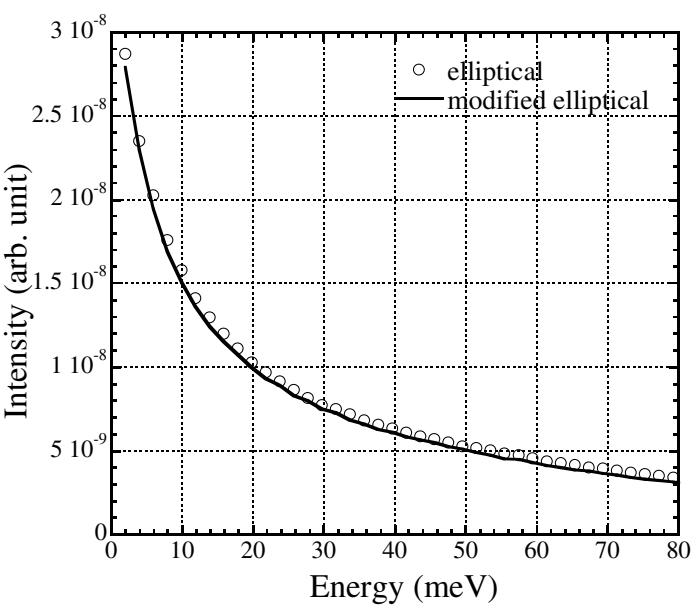

Figure 3. (a) Vertical geometries used in simulations. (b) Reflection angles at mirror when viewed from the sample position. Cases of the elliptical (solid line), modified elliptical (dashed line) and modified elliptical with "transition section" (dashed-dotted line) shapes are shown. The horizontal arrows indicate the critical angles for several values of $\mathrm{m}$ and several neutron energies. (c) Comparison of neutron flux over a $10 \times$ $20 \mathrm{~mm}^{2}$ area at the sample position. 
(a)

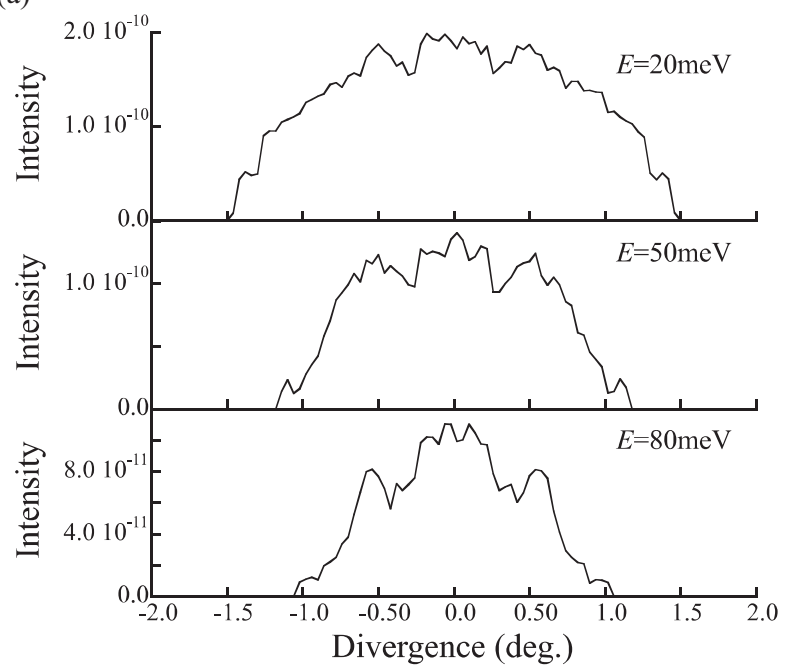

(b)

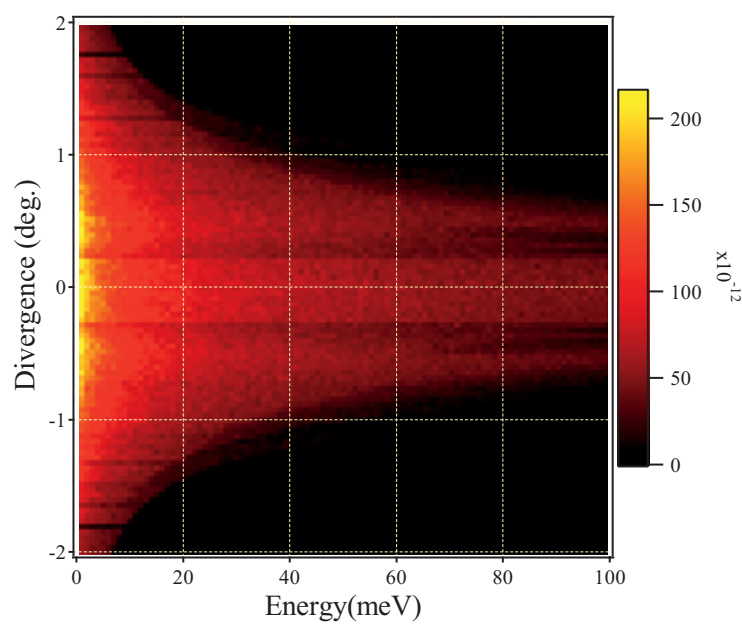

Figure 4. (a, b) Divergence in the vertical direction as a function of energy for neutrons arriving in a $10 \times 20 \mathrm{~mm}^{2}$ area at the sample position for a model without a transition section.

To eliminate this gap, we tried to avoid abrupt changes in geometry by introducing the intermediate part. This "transition section" is shown in Fig. 5a. This curve was selected to minimize the change in the curvature. By introducing a "transition section", the divergence distribution is improved as shown in Figs. 5b and c. Actually, by employing this geometry, we did not see any serious degradation of data caused by divergence anomaly, in almost five years operation of AMATERAS. The improvement in divergence distribution can be attributed to a change of the reflection angle. As one can see in Fig. 3b, the reflection angle shows a huge jump at $24 \mathrm{~m}$ from the source in the case of modified elliptical shape. This jump becomes small in the geometry using a "transition section". More sophisticated shapes for a "transition section" to diminish or completely eliminate a jump of the reflection angle would improve the performance of the beam-transport more, which should be tested in a further investigation. Therefore, our results indicate that a (a)

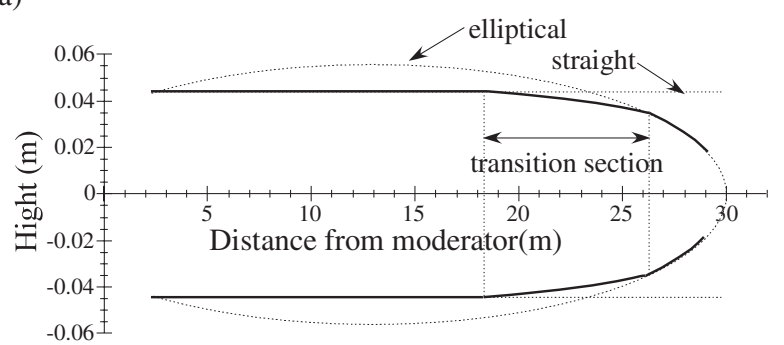

(b)

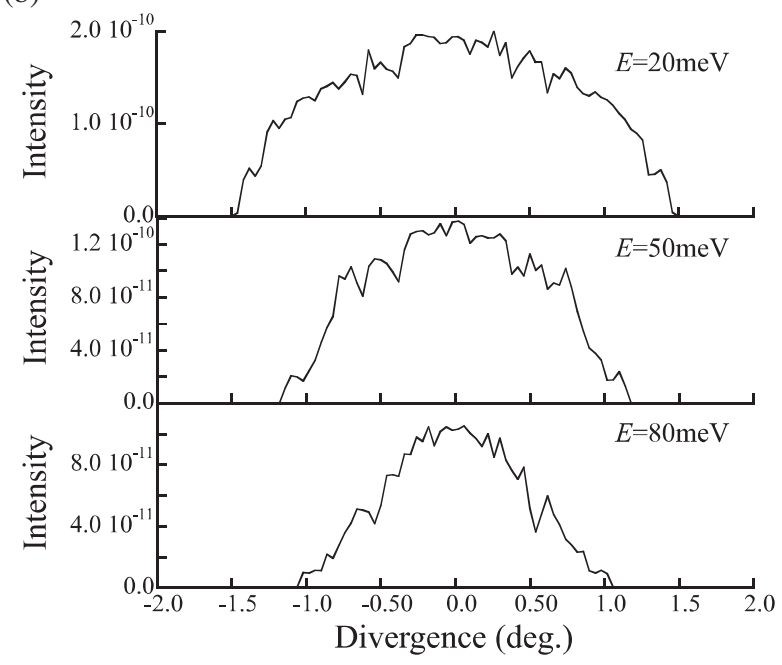

(c)

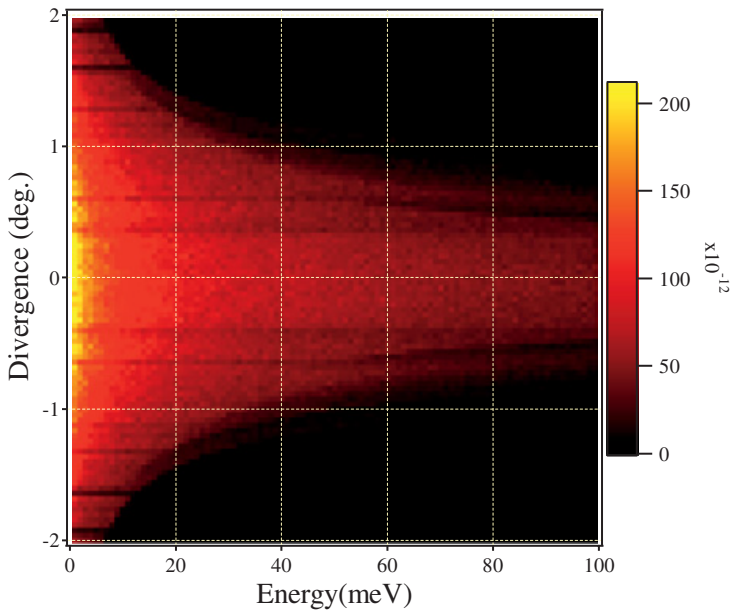

Figure 5. (a) Schematic view of a transition section. Dashed lines indicate the original elliptical line and the straight line. (b, c) divergence in the vertical direction as a function of energy for neutrons arriving in a $10 \times 20 \mathrm{~mm}^{2}$ area at the sample position for a model with a transition section.

"transition section" is generally useful for other truncated geometries.

\subsection{Focal point re-examined}

The modified elliptical shape differs significantly from the true elliptical shape. Therefore, we re-examined the position of the first focal point. 


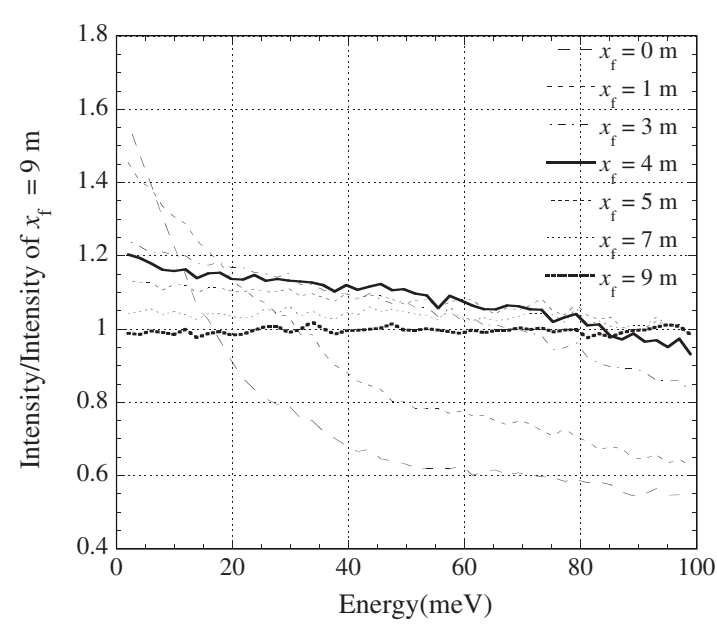

Figure 6. Energy dependencies of simulated neutron intensity of modified elliptical mirror guides with various $x_{\mathrm{f}} \mathrm{s}$, where $x_{\mathrm{f}}$ is the distance between focal point and source. Each intensity is integrated over a $10 \times 20 \mathrm{~mm}^{2}$ area at the sample position and is normalized by that of the unmodified elliptical mirror guide with $x_{\mathrm{f}}=9 \mathrm{~m}$.

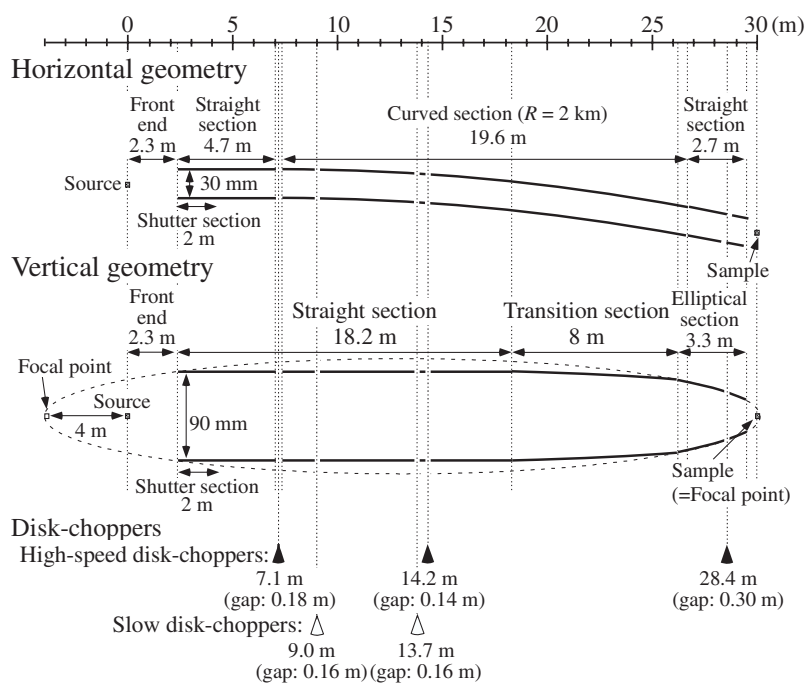

Figure 7. The final design of AMATERAS beam-transport. Positions of disk-choppers and gaps in the guide mirror to accommodate choppers are shown together. The last $1 \mathrm{~m}$ section at the downstream is not yet installed as of May 2014.

For all elliptical shapes shown in Fig. 2, we replaced the sections above $90 \mathrm{~mm}$ with straight sections and re-ran the Monte Carlo simulations. Figure 6 shows the intensity ratios for the modified elliptical mirrors with different values of $x_{\mathrm{f}}$. The intensities are normalized by those of the unmodified elliptical shape with $x_{\mathrm{f}}=9 \mathrm{~m}$, as done for Fig. 2. The geometry with $x_{\mathrm{f}}=4 \mathrm{~m}$ provides satisfactory intensity, which indicates that $x_{\mathrm{f}}$ does not need to be modified for a modified ellipsoid mirror, in the present case.

Note that the transmission rapidly degrades for $x_{\mathrm{f}} \leq$ $3 \mathrm{~m}$. This effect is considered to be attributed to the large reflection angle of the modified elliptical shapes with small $x_{\mathrm{f}}$. In other words, because the angles are not significantly affected by cutting the higher part of the mirror for large $x_{\mathrm{f}}$, modified geometries should be used when the focal point can be far from the source.
Table 1. Values of the index $m$ for the supermirrors in the AMATERAS beam transport system.

\begin{tabular}{|l|l|}
\hline Distance from the source & \multicolumn{1}{|c|}{ m } \\
\hline $2.3-4.3 \mathrm{~m}$ & Bottom \& Top 3.0, Both Sides 3.0 \\
\hline $4.3-7.0 \mathrm{~m}$ & Bottom \& Top 3.0, Both Sides 3.8 \\
\hline $7.2-18.2 \mathrm{~m}$ & Bottom \& Top 3.0, In-Side of Curve \\
& 3.0, Out-Side of Curve 3.8 \\
\hline $18.2-26.8 \mathrm{~m}$ & Bottom \& Top 3.8, In-Side of Curve \\
& 3.0, Out-Side of Curve 3.8 \\
\hline $26.8 \mathrm{~m} \sim^{* 1}$ & Bottom \& Top 3.8, Both Sides 3.8 \\
\hline
\end{tabular}

${ }^{* 1}$ The last $1 \mathrm{~m}$ section is not yet installed.

\section{Conclusions}

Based on our previous conceptual study [7], we developed a practical design for the vertical geometry of the beam-transport system of AMATERAS. An ideal elliptical mirror was modified to obtain a compromise with the requirements dictated by physical construction considerations. We replaced a part of an ellipsoidal mirror with a straight mirror without serious degradation in beam properties (intensity, divergence, and spatial distribution). These approaches were considered for AMATERAS taking into account of several constrains existing in the case of this spectrometer. Despite such circumstances, the ideas studied here can be applied also to other spectrometers in similar situations.

After optimizing the index $m$ of the mirrors, we finalized the design of the beam-transport system. In Fig. 7 and Table 1, actual design of the AMATERAS beamtransport is shown, in which positions of disk-choppers (high-speed disk choppers and slow disk-choppers) and gaps in the guide mirror which open to accommodate choppers are shown together. Although the main part of AMATERAS construction was completed in May 2009, the last $1 \mathrm{~m}$ section before the sample-is not yet installed as of May 2014.

\section{References}

[1] see http://j-parc.jp/

[2] K. Nakajima et al., J. Phys. Soc. Jpn. 80, SB028 (2011)

[3] R. Kajimoto et al., J. of Neutron Res. 16, 81 (2008)

[4] C. Shanzer et al., Nucl. Instr. and Meth. A 529, 63 (2004)

[5] L. C. Cussen et al., Nucl. Instr. and Meth. A 705, 121 (2013)

[6] M. Bertelsen et al., Nucl. Instr. and Meth. A 729, 387 (2013)

[7] R. Kajimoto et al., Physica B 385-386, 1236 (2006)

[8] K. Lefmann and K. Nielsen, Neutron News 10, 20, (1999); P. Willendrup, E. Farhi and K. Lefmann, Physica B, 350, 735 (2004)

[9] F. Mezei, in Proc. ICANS XIII, ed. G. S. Bauer and R. Becher (PSI, Villigen, 1995) 400

[10] K. Kleno et al., Nucl. Instr. and Meth. A 696, 75 (2012) 